\title{
Quasi-entropies for States of a von Neumann Algebra
}

By

\section{Dénes PETZ*}

\section{§1. Introduction}

In a general von Neumann algebra context the relative entropy of two states was defined and investigated by Araki ([3], see also [5]). When $\varphi$ and $\omega$ are normal states on a von Neuman algebra $M$ the relative entropy $S(\varphi, \omega)$ is defined by means of the relative modular operator $\Delta(\varphi, \omega)$.

$$
S(\varphi, \omega)= \begin{cases}-<\log \Delta(\varphi, \omega) \Omega, \Omega> & \text { if } s(\varphi) \geq s(\omega) . \\ +\infty & \text { otherwise. }\end{cases}
$$

where $\Omega$ is the representing vector for $\omega$ in the natural positive cone of the standard form of $\boldsymbol{M}$ and $s(\cdot)$ denotes the support of a functional. We recall that if $\boldsymbol{M}$ is finite dimensional and possesses a faithful trace $\tau$ then $\varphi(\omega)$ has a density $\rho_{\varphi}\left(\rho_{\omega}\right)$ and we have

$$
S(\varphi, \omega)=\tau\left(\rho_{\omega}\left(\log \rho_{\omega}-\log \rho_{\varphi}\right)\right) .
$$

Due to its importance in thermodynamics the relative entropy functional has been widely studied $([5],[24])$. In this paper we generalize the relative entropy functional and discuss its convexity and some other properties. Our quasi-entropy

$$
S_{f}^{k}(\varphi, \omega)
$$

depends on two parameters $k \in \boldsymbol{M}$ and a function $f:[0, \infty) \rightarrow \boldsymbol{R}$. This notion is intimately related to Lieb's concavity as Kosaki ([13]) generalized it and the $f$-divergence of Csiszár ([8], [9]) in classical information theory. We recapture Lieb's concavity in Kosaki's form

Communicated by H. Araki, October 26, 1984. Revised January 22, 1985.

* Mathematical Institute of the Hungarian Academy of Sciences, Reáltanoda u. 13-15, 1364 Budapest, PF. 127, Hungary. 
and exclude any interpolation theory. Instead, we shall use the Jensen inequality of operator concave functions. For finite dimensional algebras a part of the present paper is covered by [17]. The main convexity properties of $S_{f}^{k}(\varphi, \omega)$ will be established for arbitrary positive normal functionals but in further discussions we assume faithfulness. We mention that the discussed properties of the relative entropy follow also from the variational expression of Kosaki ([14]).

It is a pleasure to thank I. Csiszár and J. Fritz for useful conversations.

\section{§2. Relative Modular Operator}

Let $M$ be a von Neumann algebra acting on a Hilbert space $\mathscr{H}$. If $\Omega \in \mathscr{H}$ then $s^{M}(\Omega)$ denotes the smallest projection in $\mathbb{M}$ such that $s^{M}(\Omega) \Omega=\Omega$. It is easy to see that

$$
s^{M}(\Omega)=\left[M^{\prime} \Omega\right] \text {. }
$$

If $\phi, \Omega \in \mathscr{H}$ then we define a conjugate linear operator $S(\phi, \Omega)$ by the formula

$$
S(\phi, \Omega)(a \Omega+\xi)=s^{M}(\Omega) a^{*} \phi,
$$

where $a \in M$ and $\xi \in[M \Omega]^{\perp}$.

Lemma 1. $S(\phi, \Omega)$ is closable.

Proof. By the standard method one can prove that $S(\phi, \Omega) *$ is densely defined. Set

$$
F(\phi, \Omega)\left(a^{\prime} \Omega+\eta\right)=s^{M^{\prime}}(\Omega) a^{\prime *} \phi
$$

with $a^{\prime} \in \boldsymbol{M}^{\prime}$ and $\eta \in\left[\boldsymbol{M}^{\prime} \Omega\right]^{\perp}$. Since $F(\phi, \Omega) \subset S(\phi, \Omega)^{*}$ the proof is complete.

The operator $S^{*} \bar{S}$ is selfadjoint and will be called relative modular operator, in notation $S^{*} \bar{S}=\Delta(\phi, \Omega)$. We note that this definition is due to Araki (see, for example, [3] or [5]). We have $\operatorname{supp} \Delta(\phi, \Omega)$ $\subset s^{M^{\prime}}(\Omega)$ and $\Delta(\phi, \Omega) s^{M^{\prime}}(\Omega)$ is the positive selfadjoint operator associated to the closable quadratic form $a \Omega \mapsto \varphi\left(a s^{M}(\Omega) a^{*}\right)$. In other words $\Delta(\phi, \Omega)$ is the spatial derivative of $\varphi \in \mathbb{M}_{*}^{+}$with respect to $\omega^{\prime} \in\left(\boldsymbol{M}^{\prime}\right)_{*}^{+}$ 
where $\varphi(a)=\langle a \phi, \phi\rangle$ and $\omega^{\prime}\left(a^{\prime}\right)=\left\langle a^{\prime} \Omega, \Omega\right\rangle$. However, the spatial derivative is usually defined in the case when $\omega^{\prime}$ is faithful ([7], [21]).

We shall need the following observation.

Lemma 2. Let $M_{i} \subset \mathscr{B}\left(\mathscr{H}_{i}\right), \lambda, \mu>0$ and $\phi_{i}, \Omega_{i} \in \mathscr{H}_{i \cdot}$ Set $M=M_{1} \oplus \mathbb{M}_{2}, \mathscr{H}=\mathscr{H}_{1} \oplus \mathscr{H}_{2}, \Omega=\lambda \Omega_{1} \oplus \mu \Omega_{2}$ and $\phi=\lambda \phi_{1} \oplus \mu \phi_{2^{\circ}} \quad$ Then

$$
\Delta(\phi, \Omega)=\Delta\left(\phi_{1}, \Omega_{1}\right) \oplus \Delta\left(\phi_{2}, \Omega_{2}\right) \text {. }
$$

Proof. Obvious.

Araki ([3]) proved the continuity of the relative modular operator when $\phi$ and $\Omega$ are choosen from a natural positive cone If $\phi_{n} \rightarrow \phi$, $\Omega_{n} \rightarrow \Omega$ and $\Omega$ is cyclic and separating then $\Delta\left(\phi_{n}, \Omega_{n}\right) \rightarrow \Delta(\phi, \Omega)$ strongly in the generalized sense. Another kind of continuity is the following.

Lemma 3. Let $\mathbb{M} \subset \mathscr{B}(\mathscr{H})$ be a von Neumann algebra with a cyclic and separating vector $\Omega$ and $\varphi \in \mathbb{M}_{*}^{+}$. Suppose that $\left(\mathbb{H}_{n}\right)$ is an increasing sequence of von Neumann subalgebras such that $\cup_{n=1}^{\infty} \mathbb{M}_{n}$ is w-dense in $\mathbb{R}$. Let $\phi_{n} \in\left[\mathbb{M}_{n} \Omega\right]$ such that $\varphi(a)=\left\langle a \phi_{n} \phi_{n}\right\rangle$ for all $a \in \mathbb{H}_{n}$. If $\Delta_{n}$ is the relative modular operator $\Delta\left(\phi_{n}, \Omega_{n}\right)$ on $\left[\mathbb{M}_{n} \Omega\right]$ concerning $\mathbb{M}_{n}$ and $P_{n}$ denotes the projection onto $\left[\mathbb{M}_{n} \Omega\right]$ then $\Delta_{n} P_{n} \rightarrow \Delta(\phi, \Omega)$ strongly in the generalized sense.

Proof. $q_{\infty}: a \Omega \mapsto \varphi\left(a a^{*}\right)$ is a closable quadratic form and $\Delta(\phi, \Omega)$ is the associated selfadjoint operator. Similarly, $\Delta_{n}$ is the associated operator to $q_{n}=q_{\infty} \mid \mathbb{M}_{n} \Omega$. Since $\bigcup_{n=1}^{\infty} \mathbb{M}_{n}$ is strong* dense in $\mathbb{M}$ we have that $\cup_{n=1}^{\infty} \mathbb{M}_{n} \Omega$ is a core for $q_{\infty}$ and an invocation to Theorem $\mathrm{D}$ in Appendix gives the statement.

\section{§3. Definition of Quasi-entropies}

Let $M$ be a von Neumann algebra acting on a Hilbert space $\mathscr{H}$ and $\varphi, \omega$ be normal positive functionals on $\mathbb{M}$. Assume that $\varphi$ and $\omega$ have vector representatives $\phi$ and $\Omega$ in $\mathscr{H}$, that is 


$$
\varphi(a)=\langle a \phi, \phi\rangle, \omega(a)=\langle a \Omega, \Omega\rangle \quad(a \in M) .
$$

Let $f:[0, \infty) \rightarrow \mathbb{R}$ be a continuous function and $k \in \mathbb{M}$ be a fixed operator. The quasi-entropy

$$
S_{f}^{k}(\varphi, \omega)=\langle f(\Delta(\phi, \Omega)) k \Omega, k \Omega\rangle
$$

is defined by the spectral theorem. If $\int_{0}^{\infty} \lambda d E_{\lambda}$ is the spectral resolution of $\Delta(\phi, \Omega)$ then

$$
S_{f}^{k}(\varphi, \omega)=\int_{0}^{\infty} f(\lambda) d\left\|E_{\lambda} k \Omega\right\|^{2} .
$$

Of course, this integral may not exist but we need mostly the case when $f$ is concave and then $S_{f}^{k}(\varphi, \omega)$ is well-defined, however, it can be infinite. At the moment it is not clear that $S_{f}^{k}(\varphi, \omega)$ does not depend on the representing vectors but we shall come back to this point later.

Now we consider the usual relative entropy. Assume that $\varphi$ and $\omega$ are faithful normal states on $M$ and have vector representatives $\phi$ and $\Omega$ in a natural positive cone. Then

$$
S(\omega, \varphi)=-\langle\phi, \log \Delta(\omega, \varphi) \phi\rangle .
$$

Since $J \Delta(\omega, \varphi) J=\Delta(\varphi, \omega)^{-1}$ we can infer $J f(\Delta(\omega, \varphi)) J=f\left(\Delta(\varphi, \omega)^{-1}\right)$ and

$$
\begin{aligned}
S(\omega, \varphi) & =-\left\langle\Delta(\varphi, \omega)^{1 / 2} J \log \Delta(\omega, \varphi) J \Delta(\varphi, \omega)^{1 / 2} \Omega, \Omega\right\rangle= \\
& =-\left\langle\eta \Delta(\varphi, \omega) \Omega, \Omega>=-S_{\eta}^{1}(\varphi, \omega)\right.
\end{aligned}
$$

where $\eta(t)=-t \log t$. So Araki's relative entropy may be expressed as a quasi-entropy. Of course, it can be expressed without changing the arguments if we take the logarithm function but for simplicity we do not bother with non-continuous functions on $[0, \infty)$.

Another particular case is $f(t)=\sqrt{t}$. If $\varphi, \omega, \phi$ and $\Omega$ are the same as above then

$$
S_{f}^{1}(\varphi, \omega)=\left\langle\Delta(\phi, \Omega)^{1 / 2} \Omega, \Omega\right\rangle=\langle\phi, \Omega\rangle
$$

which is interpreted as a kind of transition probability and it is denoted by $P_{A}(\varphi, \omega)$. Many properties of $P_{A}(\varphi, \omega)$ follow from our results $([1],[19],[20])$.

$$
\text { If } f_{1}(t)=f(t)+a t+b \text { then }
$$

$$
S_{f_{1}}^{k}(\varphi, \omega)=S_{f}^{k}(\varphi, \omega)+a \varphi\left(k k^{*}\right)+b \omega\left(k^{*} k\right) .
$$

Hence we shall fix $f(0)$ sometimes. When $k=I$ we write simply 
$S_{f}(\varphi, \omega)$

\section{§4. Convexity Properties}

In [3] Araki proved the monotonicity of the relative entropy for special subalgebras and Uhlmann ([22]) obtained the monotonicity in the general case using quadratic interpolation techniques. In fact, he proved more: the relative entropy increases under stochastical mappings. The main result of the section is in this direction.

Theoren 4. Let $f:[0, \infty) \rightarrow \boldsymbol{R}$ be an operator monotone function with $f(0) \geq 0$. Assume that $\boldsymbol{M}_{0}$ and $\boldsymbol{M}$ are von Neumann algebras with positive normal functionals $\varphi_{0}, \omega_{0}$ and $\varphi, \omega$, respectively. If $\alpha: \mathbb{M}_{0} \rightarrow \boldsymbol{M}$ is a unit preserving 2-positive mapping such that

$$
\omega \circ \alpha \leq \omega_{0} \quad \text { and } \quad \varphi \circ \alpha \leq \varphi_{0}
$$

then for every $k \in \boldsymbol{M}_{0}$ we have

$$
S_{f}^{k}\left(\varphi_{0}, \omega_{0}\right) \geq S_{f}^{\alpha(k)}(\varphi, \omega) .
$$

Proof. Suppose that $\boldsymbol{M}\left(\boldsymbol{M}_{0}\right)$ acts on a Hilbert space $\mathscr{H}\left(\mathscr{H}_{0}\right)$ and $\varphi, \omega\left(\varphi_{0}, \omega_{0}\right)$ have vector representatives $\phi, \Omega\left(\phi_{0}, \Omega_{0}\right)$. We define linear operators $V_{\omega}, V_{\varphi}: \mathscr{H}_{0} \rightarrow \mathscr{H}$ as follows.

$$
\begin{aligned}
& V_{\omega}\left(a_{0} \Omega_{0}+\xi_{0}\right)=\alpha\left(a_{0}\right) \Omega \\
& V_{\varphi}\left(s^{M_{0}}\left(\Omega_{0}\right) a_{0} \phi_{0}+\eta_{0}\right)=s^{M}(\Omega) \alpha\left(a_{0}\right) \phi
\end{aligned}
$$

where $a_{0} \in M_{0}, \xi_{0} \in\left[M_{0} \Omega_{0}\right]^{\perp}$ and $\eta_{0} \in\left[s^{M_{0}}\left(\Omega_{0}\right) M_{0} \phi_{0}\right]^{\perp}$. We show that $V_{\omega}$ and $V_{\varphi}$ are contractions. By simple majorization we have

$$
\begin{aligned}
\left\|\alpha\left(a_{0}\right) \Omega\right\|^{2} & =\omega\left(\alpha\left(a_{0}\right) * \alpha\left(a_{0}\right)\right) \leq \omega\left(\alpha\left(a_{0}^{*} a_{0}\right)\right) \leq \omega_{0}\left(a_{0}^{*} a_{0}\right) \\
& =\left\|a_{0} \Omega_{0}\right\|^{2} \leq\left\|a_{0} \Omega_{0}+\xi\right\|^{2} .
\end{aligned}
$$

Since $\omega_{0}\left(1-s^{M_{0}}\left(\Omega_{0}\right)\right)=0$ we get $\omega\left(1-\alpha\left(s^{M_{0}}\left(\Omega_{0}\right)\right)\right)=0$. This implies $s^{M}(\Omega) \perp \operatorname{supp}\left(1-\alpha\left(s^{M_{0}}\left(\Omega_{0}\right)\right)\right)$ and $s^{M}(\Omega) \leq \alpha\left(s^{M_{0}}\left(\Omega_{0}\right)\right)$. Theorem $\mathrm{E}$ in Appendix can be applied

$$
\begin{aligned}
\left\|s^{M}(\Omega) \alpha\left(a_{0}\right) \phi\right\|^{2} & =\varphi\left(\alpha\left(a_{0}\right) *^{M}(\Omega) \alpha\left(a_{0}\right)\right) \leq \varphi\left(\alpha\left(a_{0}^{*} s^{M_{0}}\left(\Omega_{0}\right) a_{0}\right)\right) \\
& \leq \varphi_{0}\left(a_{0}^{*} s^{M_{0}}\left(\Omega_{0}\right) a_{0}\right)=\left\|s^{M_{0}}\left(\Omega_{0}\right) a_{0} \phi_{0}\right\|^{2} \\
& \leq\left\|s^{M_{0}}\left(\Omega_{0}\right) a_{0} \phi_{0}+\eta_{0}\right\|^{2}
\end{aligned}
$$


and we have arrived at $\left\|V_{\varphi}\right\| \leq 1$. From $V_{\varphi} S_{0} \subset S V_{\omega}$ we obtain

$$
V_{\varphi} \bar{S}_{0} \subset \overline{V_{\varphi} S_{0}} \subset \overline{S V_{\omega}} \subset \bar{S} V_{\omega}
$$

(Note that $\bar{S} V_{\omega}$ is closed.) Hence for any $\eta \in \mathscr{D}\left(\bar{S}_{0}\right)$ we have

$$
\left\|\bar{S} V_{\omega} \eta\right\|=\left\|V_{\varphi} \bar{S}_{0} \eta\right\| \leq\left\|\bar{S}_{0} \eta\right\| \text {. }
$$

It follows that

$$
V_{\omega}^{*} S^{*} \bar{S} V_{\omega}=\left(\bar{S} V_{\omega}\right)^{*}\left(\bar{S} V_{\omega}\right) \leq \bar{S}_{0}^{*} \bar{S}_{0}
$$

Using Theorem $\mathrm{A}$ and $\mathrm{B}$ of the Appendix we have

$$
V_{\omega}^{*} f(\Delta(\varphi, \omega)) V_{\omega} \leq f\left(V_{\omega}^{*} \Delta(\varphi, \omega) V_{\omega}\right) \leq f\left(\Delta\left(\varphi_{0}, \omega_{0}\right)\right)
$$

and in particular

$$
\left\langle V_{\omega}^{*} f(\Delta(\varphi, \omega)) V_{\omega} k \Omega_{0}, k \Omega_{0}\right\rangle \leq\left\langle f\left(\Delta\left(\varphi_{0}, \omega_{0}\right)\right) k \Omega_{0}, k \Omega_{0}\right\rangle
$$

which is equivalent to the statement.

As a consequence of Theorem $4, S_{f}^{k}(\varphi, \omega)$ does not depend on the representation in which the relative modular operator is calculated.

Corollary 5. Let $f:[0, \infty) \rightarrow \mathbb{R}$ be an operator monotone function and let $\varphi, \omega$ be normal positive functionals on the von Neumann algebra $\boldsymbol{M}$. Then

$$
S_{f}(\varphi, \omega) \leq f(\varphi(I) / \omega(I)) \omega(I) .
$$

When $M_{0}$ is a subalgebra of $\mathbb{M}$ and $k \in M_{0}$ then

$$
S_{f}^{k}\left(\varphi\left|M_{0}, \omega\right| M_{0}\right) \geq S_{f}^{k}(\varphi, \omega) .
$$

If $\varphi$ and $\omega$ are states then

$$
S_{f}(\varphi, \omega) \leq f(1)
$$

and for non-linear $f$ the equality holds if and only if $\varphi=\omega$.

Proof. (ii) is a direct consequence of Theorem 4. (ii) implies (i) since the right hand side is nothing but the quasi-entropy of the functionals restricted to the subalgebra $\boldsymbol{C} \cdot I$. To prove (iii) let $\phi_{1}-\phi_{2}$ be the Jordan decomposition of $\varphi-\omega$. If $\mathbb{M}_{0}$ is the commutative subalgebra generated by the support of $\phi_{1}$ then

$$
\|\varphi-\omega\|=\left\|\varphi_{0}-\omega_{0}\right\|
$$

where $\varphi_{0}=\varphi \mid \mathbb{M}_{0}$ and $\omega_{0}=\omega \mid \mathbb{M}_{0}$. If $f$ is not linear then $f^{\prime \prime}(1)>0([11]$, Theorem 4.5). We can use Theorem 2.1 in [8]. If $f(1)-S_{f}(\varphi, \omega)<\delta$ 
then $f(1)-S_{f}\left(\varphi_{0}, \omega_{0}\right)<\delta$ and $\left\|\varphi_{0}-\omega_{0}\right\| \leq C \sqrt{\delta}$ (for sufficiently small $\delta>0$ with a constant $C>0$ ).

Lemma 6. Let $\varphi_{1}, \varphi_{2}, \omega_{1}, \omega_{2}$ be normal positive functionals on the von Neumann algebra $\mathbf{M}$. We consider the functionals

$$
\begin{aligned}
& \varphi_{12}(a \oplus b)=\lambda \varphi_{1}(a)+\mu \varphi_{2}(b) \\
& \omega_{12}(a \oplus b)=\lambda \omega_{1}(a)+\mu \omega_{2}(b)
\end{aligned}
$$

on the algebra $\mathbb{M} \oplus \mathbb{M}$ where $\lambda, \mu>0$. Then

$$
S_{f}^{k \oplus k}\left(\varphi_{12}, \omega_{12}\right)=\lambda S_{f}^{k}\left(\varphi_{1}, \omega_{1}\right)+\mu S_{f}^{k}\left(\varphi_{2}, \omega_{2}\right) .
$$

Proof. We know from Lemma 2 that

$$
\Delta\left(\varphi_{12}, \omega_{12}\right)=\Delta\left(\varphi_{1}, \omega_{1}\right) \oplus \Delta\left(\varphi_{2}, \omega_{2}\right)
$$

and the rest follows from the definition.

Theorem 7. Assume that $f:[0, \infty) \rightarrow \mathbb{R}$ is operator monotone and $f(0)=0$. Let $\varphi_{1}, \varphi_{2}, \varphi, \omega_{1}, \omega_{2}, \omega$ be positive normal functionals on the von Neumann algebra such that

$$
\lambda \varphi_{1}+\mu \varphi_{2} \leq \varphi \text { and } \lambda \omega_{1}+\mu \omega_{2} \leq \omega_{0}
$$

If $k \in \mathbb{M}$ and $\lambda, \mu>0$ then

$$
\lambda S_{f}^{k}\left(\varphi_{1}, \omega_{1}\right)+\mu S_{f}^{k}\left(\varphi_{2}, \omega_{2}\right) \leq S_{f}^{k}(\varphi, \omega)
$$

Proof. Let $N=\mathbb{M} \oplus \mathbb{M}$ and $\varphi_{12}, \omega_{12}$ be as in Lemma 6 . So the left hand side is $S_{f}^{k \oplus k}\left(\varphi_{12}, \omega_{12}\right)$ which is smaller than $S_{f}^{k \oplus k}\left(\varphi_{12}\left|\mathbb{R} \mathbb{I}_{0}, \omega_{12}\right| \mathbb{I} \mathbb{R}_{0}\right)$ where $\mathbb{M}_{0}=\{a \oplus a: a \in \mathbb{M}\}$ is a subalgebra of $\mathbb{M}$.

Theorem 7 is a generalization of Lieb's concavity and was proved by Kosaki ([13]) by means of interpolation technique. Our method is different and based on the Jensen inequality for unbounded operators (see the Appendix).

Corollary 8. $S_{f}^{k}(\varphi, \omega)$ is jointly concave in $\varphi$ and $\omega$ under the conditions of Theorem 7.

\section{$\S 5$. Continuity $\mathbb{P}$ roperties}

Let $\mathscr{F}\left(\mathbb{M}_{*}^{+}\right)$denote the set of all faithful normal positive func- 
tionals on the algebra $M$. In this section we shall assume that $\omega \in$ $\mathscr{F}\left(M_{*}^{+}\right)$. First we use the fact that the relative modular operator is continuous if the vector representatives are taken from a natural positive cone.

Theorem 9. Let $f:[0, \infty) \rightarrow \boldsymbol{R}$ be a bounded continuous function. Assume that $\varphi_{n}, \varphi, \omega_{n}, \omega \in M_{*}^{+}$and $k_{n}, k \in M(n \in N)$ such that $\varphi_{n} \rightarrow \varphi, \omega_{n} \rightarrow \omega$ in norm and $k_{n} \rightarrow k$ strongly. If $\omega$ is faithful then

$$
S_{f}^{k}\left(\varphi_{n}, \omega_{n}\right) \rightarrow S_{f}^{k}(\varphi, \omega) \text {. }
$$

Proof. We may suppose that $f \geq 0$. So

$$
S_{f}^{k_{n}}\left(\varphi_{n}, \omega_{n}\right)=\left\|f\left(\Delta\left(\varphi_{n}, \omega_{n}\right)\right)^{1 / 2} k_{n} \Omega_{n}\right\|^{2}
$$

and $f\left(\Delta_{n}\right)^{1 / 2} k_{n} \Omega_{n}=f\left(\Delta_{n}\right)^{1 / 2} k \Omega+f\left(\Delta_{n}\right)^{1 / 2}\left(k_{n} \Omega_{n}-k \Omega\right)$. Here $f\left(\Delta_{n}\right)^{1 / 2} k \Omega \rightarrow$ $f(\Delta)^{1 / 2} k \Omega$ by the continuity of the relative modular operator and the function calculus. On the other hand $k_{n} \Omega_{n}-k \Omega \rightarrow 0$.

Cnorollary 10. Let $f:[0, \infty) \rightarrow \boldsymbol{R}$ be a continuous function bounded from below. Then

$$
(\varphi, \omega) \mapsto S_{f}^{k}(\varphi, \omega)
$$

is lower semicontinuous on $\boldsymbol{M}_{*}^{+} \times \mathscr{F}\left(\boldsymbol{M}_{*}^{+}\right)$endowed with the product of norm topologies.

Proof. There exists a sequence $\left(f_{n}\right)$ of continuous bounded functions such that $f_{n} \nearrow f$. Therefore

$$
S_{f}^{k}(\varphi, \omega)=\sup _{n} S_{f_{n}}^{k}(\varphi, \omega)
$$

is lower semicontinuous.

Theorem 11. Let $\boldsymbol{M}$ be a von Neumann algebra and $\left(\boldsymbol{M}_{n}\right)$ be an increasing sequence of subalgebras such that $\bigcup_{n=1}^{\infty} M_{n}$ is w-dense in $\boldsymbol{M}$. If $f:[0, \infty) \rightarrow \boldsymbol{R}$ is an operator convex function, $\varphi, \omega \in \mathscr{F}\left(\boldsymbol{M}_{*}^{+}\right)$and $k \in \mathbb{M}_{1}$ then

$$
S_{f}^{k}\left(\varphi_{n}, \omega_{n}\right) \rightarrow S_{f}^{k}(\varphi, \omega) \quad(n \rightarrow \infty)
$$

where $\varphi_{n}$ and $\omega_{n}$ are the restrictions of $\varphi$ and $\omega$ to $\boldsymbol{M}_{n}$. 
Proof. First we observe that by Theorem 9 the sequence $\left(S_{f}^{k}\left(\varphi_{n}, \omega_{n}\right)\right)$ is increasing. If $\Delta_{n}$ stands for the relative modular operator $\Delta\left(\varphi_{n}, \omega_{n}\right)$ then Lemma 3 tells us that $\Delta_{n} P_{n} \rightarrow \Delta(\varphi, \omega)$ strongly in the generalized sense. Hence $g\left(\Delta_{n} P_{n}\right) \rightarrow g(\Delta(\varphi, \omega))$ for every bounded continuous function. When $f$ is bounded from below then it is the increasing limit of bounded continuous functions and similarly to the previous corollary we have

$$
\underline{\lim } S_{f}^{k}\left(\varphi_{n}, \omega_{n}\right) \geq S_{f}^{k}(\varphi, \omega) .
$$

Therefore the limit of $S_{f}^{k}\left(\varphi_{n}, \omega_{n}\right)$ must be $S_{f}^{k}(\varphi, \omega)$.

If $f$ is not bounded from below then $-f$ is operator monotone and $f$ has an integral representation

$$
f(\lambda)=a+b \lambda+\int_{-\infty}^{0}(1+\lambda t)(t-\lambda)^{-1} d \mu(t)
$$

with a finite Borel measure $\mu$. Then

$$
\begin{gathered}
S_{f}^{k}\left(\varphi_{n}, \omega_{n}\right)=a \omega\left(k^{*} k\right)+b \varphi\left(k k^{*}\right)+\int_{-\infty}^{0}<\left(1+\Delta_{n} t\right)\left(t-\Delta_{n}\right)^{-1} k \Omega, k \Omega>d \mu(t) \\
\left(P_{n} k \Omega=k \Omega\right) \text { and the function } \lambda \mapsto(1+\lambda t)(t-\lambda)^{-1} \text { is bounded on }(0, \infty)
\end{gathered}
$$
so

$$
\left\langle\left(1+t \Delta_{n}\right)\left(t-\Delta_{n}\right)^{-1} k \Omega, k \Omega>\rightarrow\left\langle(1+t \Delta(\varphi, \omega))(t-\Delta(\varphi, \omega))^{-1} k \Omega, k \Omega>\right.\text { 。 }\right.
$$

On the other hand the limit is monotone and we can conclude that the integral converges.

\section{§6. Appendix}

In this part we collect some auxiliary materials we needed above. For the reader's convenience we recall definitions and results scattered in the literature and in some cases we shall slightly improve the known theorems.

$f$ always denotes a continuous function $[0, \infty) \rightarrow \mathbb{R} . \quad f$ is called operator monone if $0 \leq A \leq B$ implies $f(A) \leq f(B)$ for every bounded operators $A$ and $B$. ( $A$ and $B$ are supposed to be on the same Hilbert space and $f(A), f(B)$ are defined by the familiar functional calculi for selfadjoint operators.) For example, $t^{\alpha}(0<\alpha<1)$ is operator monotone. Every operator monotone function has a representation 


$$
f(\lambda)=a+b \lambda+\int_{-\infty}^{0}(1+\lambda t)(t-\lambda)^{-1} d \mu(t) \quad(b \geq 0)
$$

with a Borel measure $\mu$ ([2]).

We recall that if $A$ and $B$ are positive selfadjoint operators then $A \leq B$ means

(i) $\mathscr{D}\left(B^{1 / 2}\right) \subset \mathscr{D}\left(A^{1 / 2}\right)$ and $\left\|A^{1 / 2} \xi\right\| \leq\left\|B^{1 / 2} \xi\right\|$ for $\xi \in \mathscr{D}\left(B^{1 / 2}\right)$.

(ii) $(I+B)^{-1} \leq(I+A)^{-1}$.

The next theorem was proved in [4] and we repeat the proof for the sake of completeness.

Theorem A. Let $f$ be an operator monotone function on $[0, \infty)$ and $A, B$ be closed operators such that $\mathscr{D}(A) \subset \mathscr{D}(B)$ and $\|B \xi\| \leq\|A \xi\|$ for any $\xi \in \mathscr{D}(A)$. Then

$$
f\left(B^{*} B\right) \leq f\left(A^{*} A\right)
$$

Proof. The hypothesis is equivalent to the condition $B^{*} B \leq A^{*} A$. Hence we may assume that $A$ and $B$ are positive selfadjoint operators. We also suppose that $f \geq 0$. Denote $\chi_{n}$ the characteristic function of the interval $[0, n]$ and let $E_{n}=\chi_{n}(A)$ and $F_{m}=\chi_{m}(B)$. Then

$$
E_{n} B F_{m} E_{n} B F_{m} E_{n} \leq E_{n} A^{2} E_{n}
$$

and we have

$$
f\left(\left(E_{n} B F_{m} E_{n}\right)^{2}\right) \leq f\left(E_{n} A^{2} E_{n}\right)
$$

applying $f$ to bounded operators. For $\xi \in \mathscr{D}\left(f\left(A^{2}\right)^{1 / 2}\right)$ we majorize as follows.

$$
\begin{aligned}
\left\|f\left(B^{2}\right)^{1 / 2} F_{m} \xi\right\| & =\left\|f\left(\left(B F_{m}\right)^{2}\right)^{1 / 2} \xi\right\|=\lim _{n \rightarrow \infty}\left\|f\left(\left(E_{n} B F_{m} E_{n}\right)^{2}\right)^{1 / 2} \xi\right\| \\
& \leq \lim _{n \rightarrow \infty}\left\|f\left(\left(E_{n} A\right)^{2}\right)^{1 / 2} \xi\right\|=\left\|f\left(A^{2}\right)^{1 / 2} \xi\right\|
\end{aligned}
$$

Letting $m \rightarrow \infty$ the proof is completed.

The function $f:[0, \infty) \rightarrow \mathbb{R}$ is called operator convex if for any bounded positive operators $A, B \geq 0$ and $0<\lambda<1$ the inequality

$$
f(\lambda A+(1-\lambda) B) \leq \lambda f(A)+(1-\lambda) f(B)
$$

holds. Any operator monotone function is operator concave, i. e. its negative is operator convex ([2]) and any operator convex function 
is $C^{*}$-convex in the following sense. If $\sum_{i=1}^{n} C_{i}^{*} C_{i}=I$ and $A_{i} \geq 0$ is bounded $(1 \leq i \leq n)$ then the Jensen operator inequality

$$
f\left(\sum_{i=1}^{n} C_{i}^{*} A_{i} C_{i}\right) \leq \sum_{i=1}^{n} C_{i}^{*} f\left(A_{i}\right) C_{i}
$$

is satisfied (see [10], [11]). Clearly, if $f(0) \leq 0$ then the inequality holds also for degenerate $C^{*}$-convex combinations $\left(\sum_{i=1}^{n} C_{i}^{*} C_{i} \leq I\right)$. In particular, if $V$ is a contraction and $A \geq 0$ is bounded then

$$
f\left(V^{*} A V\right) \leq V^{*} f(A) V
$$

We extend this inequality to unbounded $A$.

Theoren B. Let $f$ be an operator monotone function with $f(0) \geq 0$. If $A$ is a positive selfadjoint operator and $V$ is a contraction then

$$
f\left(V^{*} A V\right) \geq V^{*} f(A) V
$$

Proof. Let $E_{n}=\chi_{n}(A)$ where $\chi_{n}$ is the characteristic function of $[0, n]$. So for $\xi \in \mathscr{D}\left(A^{1 / 2} V\right)$ we have

$$
\left\|A^{1 / 2} V \xi\right\| \geq\left\|A^{1 / 2} E_{n} V \xi\right\|
$$

and using Theorem A we obtain

$$
f\left(V^{*} A V\right) \geq f\left(V^{*} E_{n} A V\right) \text {. }
$$

Therefore

$$
f\left(V^{*} A V\right) \geq V^{*} f\left(E_{n} A\right) V=V^{*} f(A) E_{n} V+f(0) V^{*} E_{n}^{\perp} V .
$$

Letting $n \rightarrow \infty$ we arrive at the statement.

Theorem C. Let $f:[0, \infty) \rightarrow \mathbb{R}$ be an operator convex function with $f(0) \leq 0$. Then for any positive selfadjoint operator $A$ and for any contraction $V$ the inequality

$$
f\left(V^{*} A V\right) \leq V^{*} f(A) V
$$

holds.

Proof. If $f \leq 0$ then $-f$ is operator monotone ([11], Theorem 2.5) and this case is covered by Theorem B. Otherwise we set $f_{n}=\min (f, n)$. Let $A_{m}=\int_{0}^{m} \lambda d E_{\lambda}$ if $\int_{0}^{\infty} \lambda d E_{\lambda}$ is the spectral resolution of A. Since 


$$
V^{*} f\left(A_{m}\right) V \leq V^{*} f(A) V
$$

we have

$$
\left\|\left[V^{*} f\left(A_{m}\right) V+t\right]^{1 / 2} \xi\right\| \leq\left\|\left[V^{*} f(A) V+t\right]^{1 / 2} \xi\right\|
$$

where $-t$ is a lower bound of $f$. So

$$
\begin{aligned}
& \left\|\left[f_{n}\left(V^{*} A V\right)+t\right]^{1 / 2} \xi\right\|=<\left[f_{n}\left(V^{*} A V\right)+t\right] \xi, \xi> \\
& \quad=\lim _{m \rightarrow \infty}<\left[f_{n}\left(V^{*} A_{m} V\right)+t\right] \xi, \xi>\leq \lim _{m \rightarrow \infty}<\left[f\left(V^{*} A_{m} V\right)+t\right] \xi, \xi> \\
& \quad \leq\left\|\left[V^{*} f(A) V+t\right]^{1 / 2} \xi\right\|
\end{aligned}
$$

for any $\xi \in \mathscr{D}\left(\left[V^{*} f(A) V+t\right]^{1 / 2}\right)$. Letting $n \rightarrow \infty$ we get

$$
\left\|\left[f\left(V^{*} A V\right)+t\right]^{1 / 2} \xi\right\| \leq\left\|\left[V^{*} f(A) V+t\right]^{1 / 2} \xi\right\| .
$$

Now we turn to quadratic forms and convergence of selfadjoint operators (see [12]). If $q$ is a densely defined closed quadratic form on the Hilbert space $\mathscr{H}$ then there exists an associated selfadjoint positive operator $H$ such that $\mathscr{D}(q)=\mathscr{D}\left(H^{1 / 2}\right)$ and $q(\xi)=\left\|H^{1 / 2} \xi\right\|^{2}$.

Let $A_{n}$ and $A$ be positive selfadjoint operators. $A_{n} \rightarrow A$ strongly in the generalized (or resolvent) sense if $\left(I+A_{n}\right)^{-1} \rightarrow(I+A)^{-1}$ strongly as $n \rightarrow \infty$. In this case $f\left(A_{n}\right) \rightarrow f(A)$ strongly as $n \rightarrow \infty$ for any continuous bounded function $f:[0, \infty) \rightarrow \boldsymbol{R}$.

Theorem D. Let $\left(\mathscr{H}_{n}\right)$ be a sequence of closed subspaces of a Hilbert space $\mathscr{H}$. Assume that $q_{n}: \mathscr{D}_{n} \rightarrow \boldsymbol{R}$ is a densely defined closable quadratic form on $\mathscr{H}_{n}$ with the associated selfadjoint operator $H_{n}$. If $q_{\infty}$ : $\mathscr{D}_{\infty} \rightarrow \mathbb{R}$ is a closable quadratic form on $\mathscr{H}$ such that

(i) $\mathscr{D}_{n} \subset \mathscr{D}_{n+1}$ and $\cup \mathscr{D}_{n}$ is a core for $q_{\infty}$,

(ii) $q_{n}=q_{c o} \mid \mathscr{D}_{n}$

and $H_{\mathrm{c}}$ is the associated selfadjoint operator then $H_{n} P_{n} \rightarrow H_{\infty}$ strongly in the generalized sense where $P_{n}$ denotes the orthogonal projection onto $\mathscr{H}_{n}$.

Proof. Let $\mathscr{K}_{n}$ be the domain of the closure of $q_{n}$ endowed with the norm

$$
\|\xi\|_{q}=\left[\|\xi\|+\bar{q}_{\infty}(\xi)\right]^{1 / 2}
$$

$(n=1,2, \ldots, \infty)$. So $\mathscr{K}_{\text {os }}$ becomes a Hilbert space and $\left(\mathscr{K}_{n}\right)$ is an increasing sequence of closed subspaces. (i) implies that $\cup \mathscr{K}_{n}$ is dense in $\mathscr{K}_{\omega}$. Hence if $Q_{n}$ denotes the projection onto $\mathscr{K}_{n}$ then 
$Q_{n} \rightarrow I$ strongly. We set a contraction $T_{n} \in \mathscr{B}\left(\mathscr{K}_{n}\right)$ such that

$$
\left\langle T_{n} \xi, \eta\right\rangle_{q}=\langle\xi, \eta\rangle
$$

$(n=1,2, \ldots, \infty)$. Since $T_{n} Q_{n}=Q_{n} T_{\infty} Q_{n}$ we have $T_{n} Q_{n} \rightarrow T_{\infty}$ strongly. However, $T_{n} Q_{n}\left(T_{\infty}\right)$ is the restriction of $\left(I+H_{n}\right)^{-1}\left(\left(I+H_{\infty}\right)^{-1}\right)$ to $\mathscr{K}_{n}\left(\mathscr{K}_{\infty}\right)$. Using the uniform boundedness of the sequence $\left(I+H_{n}\right)^{-1}$ we obtain $\left(I+H_{n}\right)^{-1} \rightarrow\left(I+H_{\infty}\right)^{-1}$ strongly as operators in $\mathscr{B}(\mathscr{H})$.

Let $\mathscr{A}$ and $\mathscr{B}$ be $C^{*}$-algebras. We recall that the linear mapping $\alpha: \mathscr{A} \rightarrow \mathscr{B}$ is 2-positive if $\alpha \otimes$ id: $\mathscr{A} \otimes M_{2} \rightarrow \mathscr{B} \otimes M_{2}$ is a positive mapping. Assume that $\alpha: \mathscr{A} \rightarrow \mathscr{B}$ is a 2-positive unit preserving mapping and $T \in \mathscr{A}_{+}$is invertible. Then

$$
\alpha\left(S^{*} T^{-1} S\right) \geq \alpha\left(S^{*}\right) \alpha(T)^{-1} \alpha(S)
$$

for any $S \in \mathscr{A}$ (see [6], Proposition 4.1). In fact, the above inequality is equivalent to the 2-positivity of $\alpha$.

Theorem E. Let $\mathscr{A}_{0}$ and $\mathscr{A}$ be $C^{*}$-algebras and let $\alpha: \mathscr{A}_{0} \rightarrow \mathscr{A}$ be a unit preserving 2-positive mapping. Suppose that $q \in \mathscr{A}_{0}$ and $p \in \mathscr{A}$ are projections such that $p \leq \alpha(q)$. Then for any $a \in \mathscr{A}_{0}$ the inequality

$$
\alpha(a) * p \alpha(a) \leq \alpha\left(a^{*} q a\right)
$$

holds.

Proof. First we note that $p$ commutes with $\alpha(q)$. Indeed, $p \leq \alpha(q) \leq I$ implies $\alpha(q) \xi=\xi$ wherever $p \xi=\xi$.

Let $C_{\lambda}=q+\lambda(I-q)$. Since $C_{\lambda}$ is invertible for $\lambda>0$ we have

$$
\alpha(a)^{*} \alpha\left(C_{\lambda}\right)^{-1} \alpha(a) \leq \alpha\left(a^{*} C_{\lambda}^{-1} a\right) .
$$

When $\lambda \rightarrow \infty$ then $C_{\lambda}^{-1} \rightarrow q$ in norm and the right hand side tends to $\alpha\left(a^{*} q a\right)$. On the other hand, $\alpha\left(C_{\lambda}\right)^{-1} \geq p$ since $\alpha(q)$ and $p$ are commuting operators.

\section{References}

[1] Alberti, P.M. and Uhlmann A., Transition probabilities on $\mathrm{W}^{*_{-}}$and $\mathrm{C}^{*_{-} \text {-algebras, }}$ Proceedings of the Second Int. Conf. on Operator Algebras, Ideals and Their Appl. in Theor. Physics, 5-11, (Teubner-Texte zur Math. 67, Teubner Verlag, Leipzig, 1984).

[2] Ando, T., Topics on operator inequalities, Hokkaido Univ., Sapporo, 1978.

[3] Araki, H., Relative entropy of a von Neumann algebra I, II, Publ. RIMS Kyoto Univ., 11 (1976), 809-833 and 13 (1977), 173-192. 
[4] Araki, H. and Masuda, T., Positive cones and $L_{p}$-spaces for von Neumann algebras, Publ. RIMS Kyoto Univ., 18 (1982), 339-441.

[5] Bratteli, O, and Robinson, D. V., Operator algebras and quantum statistical mechanics II, Springer Verlag, Berlin, 1981.

[6] Choi, M. D., Some assorted inequalities for positive linear maps on $C^{*}$-algebras, $J$. Operator Theory, 4 (1980), 271-285.

[7] Connes, A., On a spatial theory of von Neumann algebras, J. Functional Analysis, 35 (1980), 153-164.

[8] Csiszár, I., Measures of information type for difference of probability distributions (in Hungarian), (C. Sc. Thesis, Budapest, 1966).

[9] , Information-type measures of difference of probability distributions and indirect observations, Studia Sci. Math. Hungar., 2 (1967), 299-318.

[10] Davis, C., Notions generalizing convexity for functions defined on spaces of matrices, Proc. Amer. Math. Soc. Symposia (Gonvexity), 7 (1963), 187-201.

[11] Hansen, F. and Pedersen, G. K., Jensen's inequality for operators and Löwner's theorem, Math. Ann., 258 (1982), 229-241.

[12] Kato, T., Perturbation theory of linear operators, Springer Verlag, Berlin, 1966.

[13] Kosaki, H., Interpolation theory and the Wigner-Yanase-Dyson-Lieb concavity, Commun. Math. Phys., 87 (1982), 315-329.

[14] - Variational expressions of relative entropy of states on $W^{*}$-algebras, (Preprint, 1984).

[15] Lieb, E. H., Convex trace functions and the Wigner-Yanase-Dyson conjecture, Adv. in Math., 11 (1973), 267-288.

[16] Petz, D., Properties of the relative entropy of states of a von Neumann algebra, to appear in Acta Math. Hungar.

[17] , Quasi-entropies for finite quantum systems, to appear in Rep. Math. Phys.

[18] Pusz, W. and Woronowicz, S., Form convex functions and WYDL and other inequalities, Lett. Math. Phys., 2 (1978), 505-512.

[19] Raggio, G. A., Comparison of Uhlmann's transition probability with the one induced by the natural positive cone of a von Neumann algebra in standard form, Lett. Math. Phys., 6 (1982), 233-236.

[20] , Generalized transition probabilities and applications, Quantum Probability and Appl. to the Quantum Theory of Irr. Proc., 327-335, (Lecture Notes in Math., 1055, Springer Verlag, Berlin, 1984).

[21] Strătilă, S.., Modular theory of operator algebras, Abacus Press, Tunbridge Wells, 1981.

[22] Uhlmann, A., Relative entropy and the Wigner-Yanase-Dyson-Lieb concavity in an interpolation theory, Commun. Math. Phys., 54 (1977), 21-32.

[23] Umegaki, H., Conditional expectation in an operator algebra IV (entropy and information), Kodai Math. Sem. Rep., 14 (1962), 59-85.

[24] Wohrl, A., General properties of entropy, Rev. Modern Phys., 50 (1978), 221-260. 